\title{
GlueX Experiment at Jefferson Lab
}

\author{
Sean Dobbs*t \\ Northwestern University \\ E-mail: s-dobbs@northwestern.edu
}

The GlueX experiment at Jefferson Lab is a fixed target photoproduction experiment located in the newly-constructed Hall D. It is designed to study the spectroscopy of a variety of hadrons, with special emphasis on hybrid mesons. The experiment uses a $12 \mathrm{GeV}$ electron beam incident on a diamond radiator to produce a linearly-polarized tagged bremsstrahlung photon beam of energies up to $12 \mathrm{GeV}$, peaking at $9 \mathrm{GeV}$. This photon beam is incident on a liquid hydrogen target surrounded by a spectrometer that combines charged particle tracking, electromagnetic calorimetry, and time-of-flight measurements . Initial commissioning data were taken at lower beam energies in the Fall of 2014 and Spring of 2015, with a final commissioning run at full energy scheduled in the Spring of 2016. Results for detector performance and preliminary results for the reconstruction of several exclusive final states are presented.

38th International Conference on High Energy Physics 3-10 August 2016

Chicago, USA

\footnotetext{
* Speaker.

${ }^{\dagger}$ On behalf of the GlueX Collaboration.
} 


\section{Introduction}

The GlueX Experiment [1] is the flagship experiment of the newly-constructed Hall D at the Thomas Jefferson National Accelerator Facility (JLab), a world-leading nuclear physics research facility funded by the U.S. Department of Energy's Office of Science, located in Newport News, VA. The GlueX Collaboration consists of over 120 members from 24 institutions, with more joining.

The primary goal of GlueX is to advance the quantitative understanding of confinement in Quantum Chromodynamics (QCD). In particular, a deeper understanding of the soft gluon field responsible for confinement is needed. "Hybrid" mesons [2], those with explicit gluonic degrees of freedom, are ideal states for studying these gluonic fields, and we aim to firmly establish these states and to map out their spectrum [3]. The large, precise data set that will be collected can also be used to make measurements of strangeonia and other light meson states, strange-quark-containing baryons, and charm production near threshold.

The GlueX detector measures photoproduction events generated by a photon beam incident on a $30 \mathrm{~cm}$ long liquid hydrogen target. The photon beam is generated by a $12 \mathrm{GeV}$ electron beam incident on a diamond radiator, which generates a linearly-polarized beam of photons with $9 \mathrm{GeV}$ peak energy via coherent bremsstrahlung. The scattered electrons are measured in the tagger spectrometer to "tag" the photon energies. The Pair Spectrometer and Triplet Polarimeter monitor the photon flux and polarization using well-known QED processes. The degree of linear polarization in the peak energy range has been measured to be $40 \%$.

The main detector, illustrated in Fig. 1 consists of a $2 \mathrm{~T}$ solenoid magnet with the Central and Forward Drift Chamber (CDC \& FDC) for charged particle, a lead/scintillating fiber Barrel Calorimeter (BCAL) and lead-glass Forward Calorimeter (FCAL) for neutral particle detection,

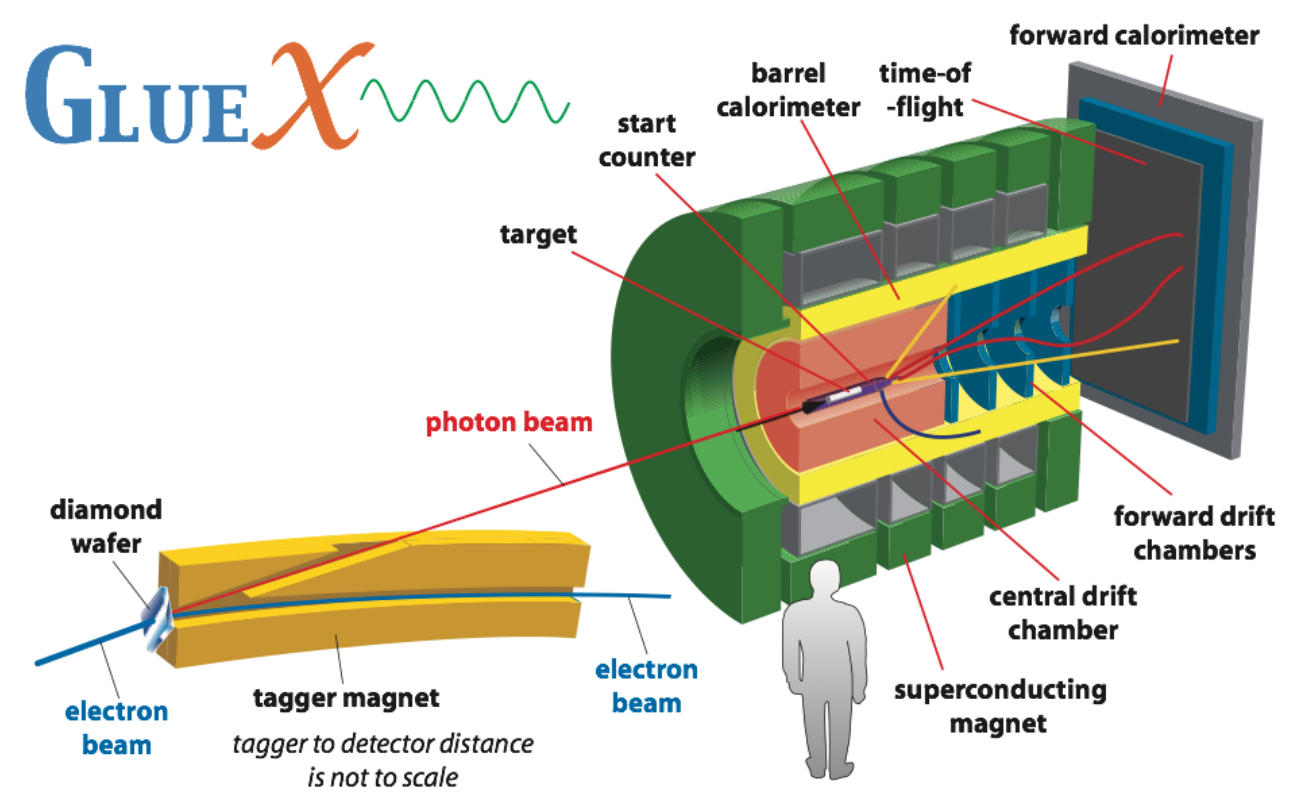

Figure 1: Diagram of the GlueX Detector 

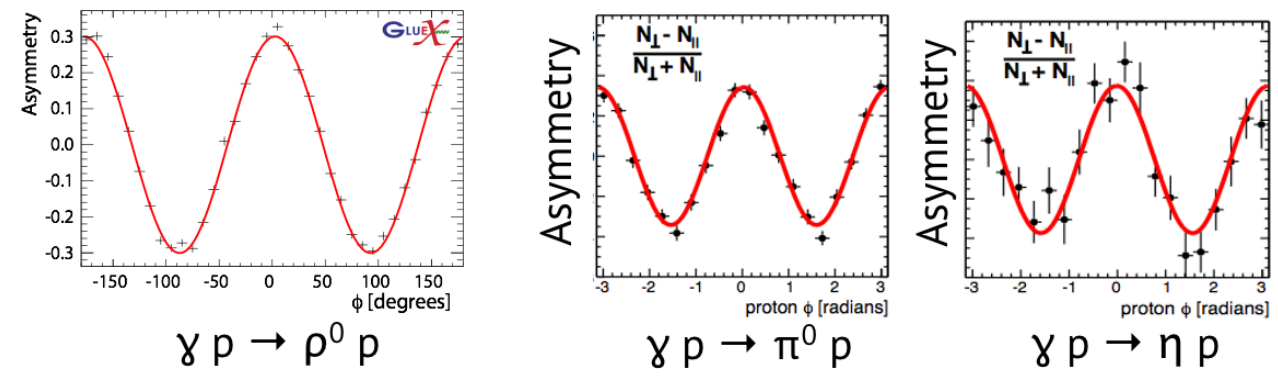

Figure 2: Examples of preliminary results for single meson production asymmetries.

and a Start Counter (SC) and forward Time-of-Flight wall (TOF) for precision timing measurements. A Cherenkov detector for enhanced particle identification using components from the BaBar DIRC is expected to be installed for running in 2018/19.

\section{Physics Goals \& Early Measurements}

QCD describes the interaction of quarks and gluons, and predicts the spectrum of $q \bar{q}$ mesons and $q q q$ baryons. There should also be "hybrid" mesons where the gluons contributes directly to the spin-parity of the state. These hybrids can be identified by having $J^{P C}$ values not accessible to normal $q \bar{q}$ mesons.

The first measurements in GlueX will be of polarization and beam asymmetry measurements of pseudoscalar and vector mesons, leading to spin-density matrix element analyses to understand the production mechanisms involved. Cross section measurements will then be made, followed by amplitude analyses of known mesons and searches for exotic mesons. Full understanding of this data will require close collaboration with theorists.

The beam asymmetry $\Sigma$ gives insight into the production mechanism of a given reaction, and one of the first measurements to make is that of the beam asymmetry of single pseudoscalar and vector mesons. With a subset of the data taken in Spring 2016, we have measured the experimental asymmetry for $\gamma p \rightarrow \rho^{0} p, \rho^{0} \rightarrow \pi^{+} \pi^{-}$with more than 1000 times the events measured in the old SLAC bubble chamber experiments at $E_{\gamma} \sim 9 \mathrm{GeV}$. We have also performed similar measurements in $\gamma p \rightarrow\left(\pi^{0}, \eta\right) p, \pi^{0}, \eta \rightarrow \gamma \gamma$, which will be used to test theoretical models. These preliminary results are illustrated in Fig. 2. The beam polarization $(P)$ measurements needed to extract $\Sigma$ are still being finalized.

We expect that this data will yield many other spectroscopic results, and present two examples under analysis. We see a peak at $\sim 1.6 \mathrm{GeV}$ in the $\pi^{+} \pi^{-}$mass spectrum of $\gamma p \rightarrow \pi^{+} \pi^{-} p$ (see Fig. 2, left panel), confirming SLAC?s observation in this reaction. In $\gamma p \rightarrow 4 \gamma p$ (see Fig. 2, right panel), we see several states decaying to $\pi^{0} \pi^{0}$ and $\eta \pi^{0}$.

\section{Summary}

The GlueX experiment has been successfully commissioned and is ready for the start of its first physics run in Fall 2016. Initial physics results are being prepared. The addition of improved kaon 

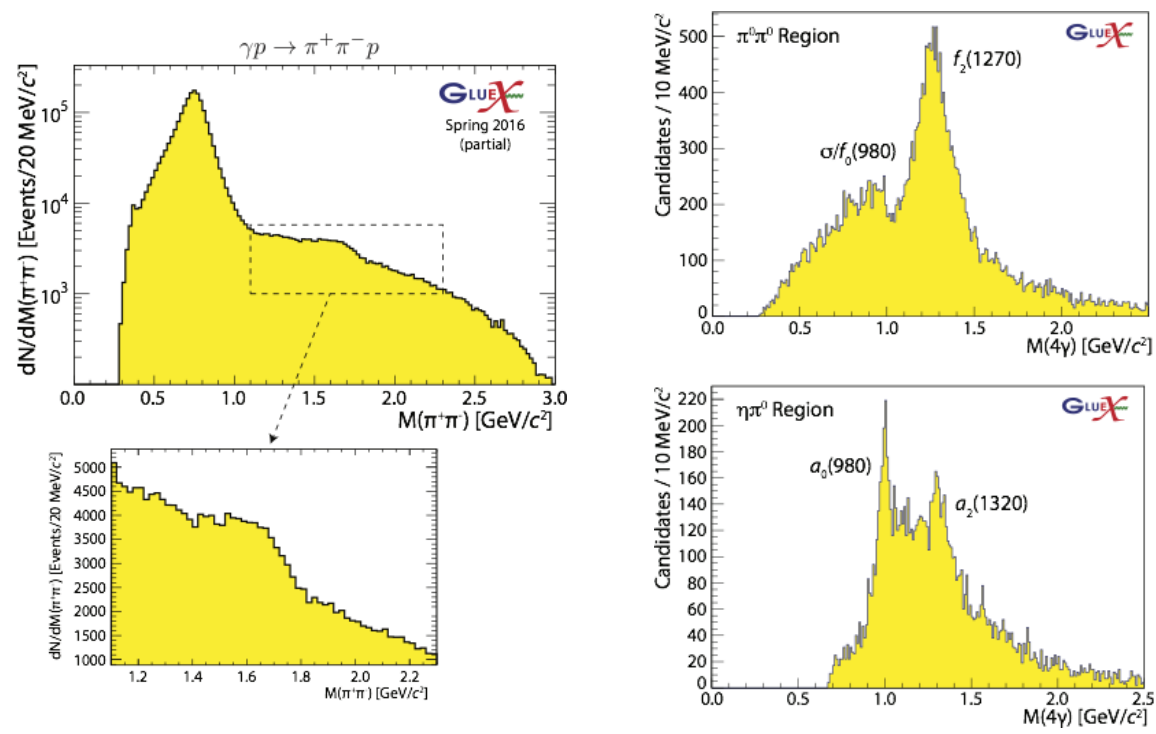

Figure 3: Examples of mass spectra from a fraction of the Spring 2016 GlueX data. (Left) $\pi^{+} \pi^{-}$invariant mass spectra, with an inset illustrating the enhancement at $1.6 \mathrm{GeV}$. (Right) $\pi^{0} \pi^{0}$ and $\eta \pi^{0}$ mass spectra, illustrating the $a$ and $f$ states strongly excited in this data.

identification and higher luminosity in 2018 will allow a full exploration of the light quark meson sector. Other planned GlueX measurements include the eta lifetime, charged pion polarizability, and rare eta decays, and charm-quark containing hadrons.

\section{References}

[1] For more details of the experiment, see H. Al Ghoul et al. [GlueX Collaboration], AIP Conf. Proc. 1735, 020001 (2016), and references therein.

[2] C. A. Meyer and E. S. Swanson, Prog. Part. Nucl. Phys. 82, 21 ?58 (2015).

[3] J. J. Dudek, R. G. Edwards, P. Guo, and C. E. Thomas [Hadron Spectrum], Phys. Rev. D 88, 094505 (2013). 